\title{
ARTICLE OPEN Spin coherence in two-dimensional materials
}

\author{
Meng Ye $\mathbb{D}^{1}$, Hosung Seo ${ }^{1,2,3,4}$ and Giulia Galli ${ }^{1,2,5,6}$
}

Spin defects in semiconducting solids are promising platforms for the realization of quantum bits. At low temperature and in the presence of a large magnetic field, the central spin decoherence is mainly due to the fluctuating magnetic field induced by nuclear spin flip-flop transitions. Using spin Hamiltonians and a cluster expansion method, we investigate the electron spin coherence of defects in two-dimensional (2D) materials, including delta-doped diamond layers, thin Si films, MoS 2 , and h-BN. We show that isotopic purification is much more effective in $2 \mathrm{D}$ than in three-dimensional materials, leading to an exceptionally long spin coherence time of more than $30 \mathrm{~ms}$ in an isotopically pure monolayer of $\mathrm{MoS}_{2}$.

npj Computational Materials (2019)5:44; https://doi.org/10.1038/s41524-019-0182-3

\section{INTRODUCTION}

Deep-level defects in wide-bandgap semiconductors exhibit localized electronic energy levels with paramagnetic spin states, and hence they constitute promising platforms for quantum bits (qubits). ${ }^{1}$ Defect-based solid-state spin qubits have been widely studied, including the nitrogen-vacancy (NV) center in diamond, ${ }^{2,3}$ vacancies in $\mathrm{SiC}_{1}^{4-7}$ donor spins in silicon, ${ }^{8}$ and rare-earth ions, ${ }^{9}$ targeting quantum computing, and sensing applications. ${ }^{10,11}$ The spin coherence time $T_{2}$, usually measured by Hahn-echo experiments, ${ }^{12}$ is a critical property for qubit applications. ${ }^{13}$ In quantum computers, in order to achieve quantum error correction, at least $10^{4}$ quantum gate operations need to be performed within $T_{2}$, yielding a desired coherence time larger than $100 \mu \mathrm{s} .{ }^{13,14}$ The sensitivity of quantum sensors, which is proportional to $\frac{1}{\sqrt{T_{2}}}, 15$ benefits as well from qubits with long coherence times.

The decoherence of spin qubits can arise from different sources, including the magnetic noise from high-density paramagnetic defects and dangling bonds on the material surface. ${ }^{16,17}$ In highly purified bulk solids, the phase decoherence is mainly caused by the entanglement between the electron spin qubit and the nuclear spin bath of the host material. ${ }^{18}$ Thus, isotopic purification is an effective technique to enhance $T_{2} \cdot{ }^{19,20}$ For example, the ensemble $T_{2}$ of the NV center in diamond has been extended from $0.63 \mathrm{~ms}^{21}$ to $1.8 \mathrm{~ms}^{19}$ by reducing the concentration of ${ }^{13} \mathrm{C}$ nuclear spins from 1.1 to $0.3 \%$. In general, solids with a low concentration of nuclear spins are desirable spin qubits hosts. ${ }^{1}$

Two-dimensional (2D) materials exhibit a variety of promising properties for the realization of hosts of defect-based spin qubits, including their facile transfer and integration into hybrid quantum systems. $^{22-25}$ In 2D systems, point defects can be created and precisely located using a scanning transmission electron microscope or an annular dark field microscopy. ${ }^{26}$ Furthermore, compared with thin films of three-dimensional (3D) solids, 2D layers are free from unsaturated dangling bonds at the surface that are detrimental to spin coherence. However, the nuclear spin environments in many 2D materials are much more complex than, for example, in diamond.
In this work, we investigate the coherence of spin qubits in 2D materials and propose strategies to search for ideal host materials. We first explore the effect of dimensionality, crystal geometry, and nuclear spin moment on the spin coherence. Especially, we discuss the interplay of dimensionality and nuclear spin concentration in determining $T_{2}$. We then predict $T_{2}$ in single layer and multi-layer $\mathrm{MoS}_{2}$ and $\mathrm{h}-\mathrm{BN}$, which are among the most studied 2D host candidates, $27-35$ and we propose design rules to further improve the spin coherence and obtain spin qubits with a performance superior to that, for example, of 3D materials such as diamond and SiC.

\section{RESULTS AND DISCUSSION}

Theoretical framework

We describe an electron spin $\boldsymbol{S}$ immersed in a nuclear spin bath using a central spin Hamiltonian $H=H_{\mathrm{S}}+H_{\mathrm{B}}+H_{\mathrm{S}-\mathrm{B}}$, where $H_{\mathrm{S}}$ and $H_{B}$ represent the electron spin and the nuclear spin bath, respectively, and $H_{S-B}$ describes the qubit-bath interaction: ${ }^{36-39}$

$$
\begin{aligned}
& H_{\mathrm{S}}=-\gamma^{\mathrm{e}} \boldsymbol{B} \cdot \boldsymbol{S}+\boldsymbol{S} \cdot \boldsymbol{D} \cdot \boldsymbol{S}, \\
& H_{\mathrm{B}}=-\boldsymbol{B} \cdot \sum_{i} \gamma_{i}^{\mathrm{n}} \boldsymbol{I}_{i}+H_{\mathrm{nn}}, \\
& H_{\mathrm{nn}}=\frac{\mu_{0}}{4 \pi} \sum_{i<j} \gamma_{i}^{\mathrm{n}} \gamma_{j}^{\mathrm{n}} \frac{\boldsymbol{I}_{i} \cdot \boldsymbol{I}_{j}-3\left(\boldsymbol{I}_{i} \cdot \widehat{\boldsymbol{r}}_{i j}\right)\left(\boldsymbol{I}_{j} \cdot \widehat{\boldsymbol{r}}_{i j}\right)}{r_{i j}^{3}}, \\
& H_{\mathrm{S}-\mathrm{B}}=\boldsymbol{S} \cdot \sum_{i} \boldsymbol{A}_{i} \cdot \boldsymbol{I}_{i},
\end{aligned}
$$

where $\gamma^{\mathrm{e}}$ and $\gamma^{\mathrm{n}}$ are the gyromagnetic ratios of electron and nuclear spins, and $r_{i j}$ is the distance between the nuclear spins $\boldsymbol{I}_{i}$ and $\boldsymbol{I}_{j} . H_{\mathrm{S}}$ describes the Zeeman splitting of the spin sublevels due to an applied magnetic field $(\boldsymbol{B}=B \widehat{\boldsymbol{z}})$ and $\boldsymbol{D}$ is the zero-field splitting, different from zero for $S>\frac{1}{2}$. In the case of a large level splitting arising from $H_{S}$, the electron spin $S_{Z}$ is a good quantum number. $H_{\mathrm{B}}$ is the sum of the Zeeman energy and the dipolar

\footnotetext{
${ }^{1}$ Institute for Molecular Engineering, The University of Chicago, Chicago, IL, USA; ${ }^{2}$ Materials Science Division, Argonne National Laboratory, Lemont, IL, USA; ${ }^{3}$ Department of

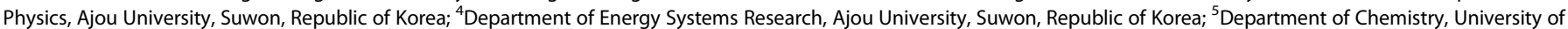
Chicago, Chicago, IL, USA and ${ }^{6}$ Institute for Molecular Engineering, Argonne National Laboratory, Lemont, IL, USA

Correspondence: Giulia Galli (gagalli@uchicago.edu)
}

Received: 4 December 2018 Accepted: 25 March 2019

Published online: 09 April 2019 
interaction of nuclear spins. The latter describes a fluctuating magnetic noise present in the system, which is entangled with the electron spin through the hyperfine interaction entering $\mathrm{H}_{\mathrm{S}-\mathrm{B}}{ }^{40}$

The hyperfine interaction between a nuclear and an electron spin consists of two parts, the Fermi contact and the dipolar interaction. Because of the localized nature of the spin density of deep center defects, only a small number of nuclear spins near the spin qubit contributes to the Fermi contact, which is thus considered negligible and not included in our calculations. In addition, as $S_{z}$ is a good quantum number in the presence of a strong magnetic field or a large zero-field splitting, the $S_{x}$ and $S_{y}$ terms are neglected in $H_{\mathrm{S}-\mathrm{B}}$.

\section{Effect of reduced dimensionality}

We first focused on the effect of reduced dimensionality (from 3D to $2 \mathrm{D}$ materials) on the $T_{2}$ value, and we considered host materials with only one nuclear spin species. The NV center in diamond, where the ground state spin $|0\rangle$ and $|1\rangle$ states of the defect are used as a two-level system, is an ideal example as only ${ }^{13} \mathrm{C}$ contributes to the decoherence. We investigated the variation of $T_{2}$ as a function of the thickness of a diamond slab. In practice, a diamond thin slab with tunable ${ }^{13} \mathrm{C}$ concentration could be obtained through delta-doping a thin layer of ${ }^{13} \mathrm{C}$ nuclear spins embedded in a 3D nuclear-spin-free diamond matrix. ${ }^{41}$ In order to approach the 2D limit, we also considered graphite as a model host material with a NV-like spin state localized at a C-vacancy site. Although a NV-like spin qubit in graphite is hypothetical, we note that spin qubits have recently been realized as edge states of graphene nanoribbons. $^{42}$

We placed a spin qubit in the center of a thin diamond slab of width $d$ or in the central layer of a $n$-layer graphite (see Supplementary Information) and we considered various nuclear spin concentration. We applied a magnetic field $B=0.05 \mathrm{~T}$ along the three-fold axis of the defect; in this direction $T_{2}$ attains its maximum value. ${ }^{21,38}$ The chosen value of $B$ corresponds to a strong field under which $T_{2}$ reaches saturation and the dominant decoherence mechanism arises from nuclear spin flip-flop transitions. ${ }^{43,44}$

Our predicted coherence times for diamond thin slabs and for multi-layer graphites are plotted in Fig. 1 as a function of the ${ }^{13} \mathrm{C}$ nuclear spin concentration $\eta$. The values $a$ and $\beta$ obtained by fitting the log-log plot in Fig. 1 with $T_{2}=a \eta^{-\beta}$ are summarized in Table 1 . The figure and the table show that reducing the slab thickness can effectively enhance $T_{2}\left(a_{2 \mathrm{D}}>a_{\text {slab }}>a_{3 \mathrm{D}}\right)$. This finding is not surprising since the nuclear spin density is reduced in $2 \mathrm{D}$.

(a)

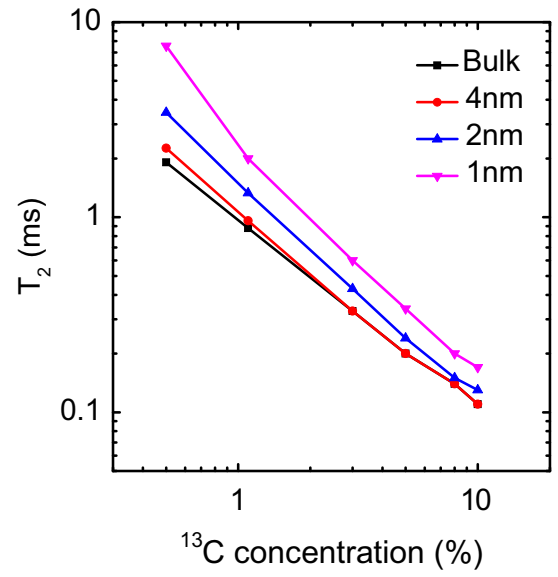

Most importantly, our results show that the enhancement of $T_{2}$ relative to the bulk is more prominent at lower nuclear spin concentrations $\left(\beta_{2 \mathrm{D}}>\beta_{\text {slab }}>\beta_{3 \mathrm{D}}\right)$. We have also clearly identified the thickness of the slab at which $T_{2}$ is significantly enhanced, which turns out to be few nanometers. The interplay between the nuclear spin concentration and the slab thickness can be understood as follows. The major contributions to decoherence originate from nuclear spins belonging to a region of radius $R$ centered on the spin qubit and $R$ decreases as the concentration increases. For example, in bulk diamond, we found that $R \approx 5 \mathrm{~nm}$ for $1.1 \%{ }^{13} \mathrm{C}$, and it reduces to $3 \mathrm{~nm}$ for $10 \%{ }^{13} \mathrm{C}$, and to $1.5 \mathrm{~nm}$ for $100 \%{ }^{13} \mathrm{C}$. A major increase of $T_{2}$ due to a decrease of the slab thickness $d$ is expected only if $d \ll R$; hence, for thin slabs the enhancement of $T_{2}$ is larger when the nuclear spin concentration is lower.

\section{Effect of crystal geometry}

Crystal geometry, in particular the interatomic distance, has a significant impact in determining $T_{2}$, since the energy scale of nuclear spin flip-flop transitions is proportional to $\frac{1}{r^{3}}$ (see Eq. 3). ${ }^{44,45}$ An example is shown in Fig. 1: the $T_{2}$ of a bulk graphite is always larger than that of a bulk diamond, due to the larger average interatomic distance in graphite. Silicon has the same lattice structure as carbon diamond, but with a much larger lattice constant. In addition, deep-level defects have been observed in Si as potential spin qubits. ${ }^{46,47}$ For a localized defect in Si with the same spin states as a NV center, for example, a tin vacancy, ${ }^{47}$ we predict $T_{2}$ in the bulk and in a 4-nm slab to be 1.15 and $1.25 \mathrm{~ms}$, respectively, for $4.7 \%$ of ${ }^{29} \mathrm{Si}$, values that are about $35-30 \%$ larger than the corresponding ones in $\mathrm{C}$ diamond with $1.1 \%$ of ${ }^{13} \mathrm{C}$. This points at

Table 1. Computed coherence time $T_{2}=a \eta^{-\beta}$ of diamond and graphite, as obtained by fitting the curves shown in Fig. 1, where $\eta$ is the ${ }^{13} \mathrm{C}$ concentration

\begin{tabular}{lllllllll}
\hline & \multicolumn{3}{l}{ Diamond } & & & \multicolumn{3}{l}{ Graphite } \\
\cline { 2 - 4 } \cline { 8 - 10 } & Bulk & $4 \mathrm{~nm}$ & $2 \mathrm{~nm}$ & $1 \mathrm{~nm}$ & & Bulk & 3-layer & 1-layer \\
\hline$a(\mathrm{~ms})$ & 0.97 & 1.07 & 1.52 & 2.66 & & 1.38 & 3.99 & 12.56 \\
$\beta$ & 0.95 & 1.01 & 1.11 & 1.25 & & 0.99 & 1.35 & 1.57 \\
\hline
\end{tabular}

The tables shows the parameters $a(\mathrm{~ms})$ and $\beta$. The constant $a$ is equal to the $T_{2}$ value at $1 \%{ }^{13} \mathrm{C}$ concentration, while $\beta$, which is the slope in Fig. 1, represents the sensitivity of $T_{2}$ to nuclear spin concentration.

(b)

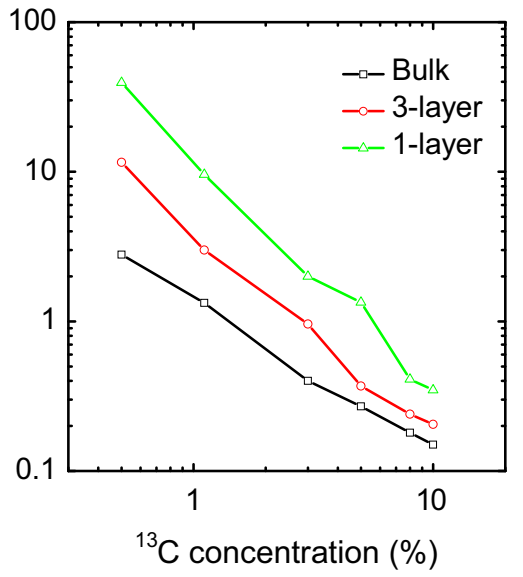

Fig. 1 Computed coherence time $T_{2}$ as a function of ${ }^{13} \mathrm{C}$ concentration for bulk and thin films diamond (left panel) and for bulk and thin layers graphite (right panel). $T_{2}$ is predicted to be $0.88 \mathrm{~ms}$ for nitrogen-vacancy (NV) center in bulk diamond at the nuclear spin natural abundance $\left(1.1 \%{ }^{13} \mathrm{C}\right)$, in agreement with previous reports ${ }^{38,44,48,49}$ 
thin Si slabs as potentially excellent host materials for qubits, since quantum confinement is expected to increase the band gap of $\mathrm{Si}$, possibly to desired values to create stable spin-defects within the gap.

\section{Effect of large nuclear spins}

So far, we only considered a nuclear spin $I=\frac{1}{2}$, such as ${ }^{13} \mathrm{C}$ and ${ }^{29} \mathrm{Si}$. As most nuclei of $2 \mathrm{D}$ materials have $I>\frac{1}{2}$, we qualitatively investigated the dependence of $T_{2}$ on $I$, by arbitrarily varying $I$ of ${ }^{13} \mathrm{C}$ from $\frac{1}{2}$ to $\frac{9}{2}$ in our bulk diamond calculations. We found that the coherence time is approximately inversely proportional to I (in particular $\left.T_{2}(I) / T_{2}\left(I=\frac{1}{2}\right)=-0.045+0.522 / I\right)$ and the result can be rationalized as follows. The decoherence is determined by the strength of nuclear spin flip-flop transitions and the number of transition channels. In a volume $V$ with $N$ nuclear spins, the number of nuclear spin pairs is $N_{\text {pair }}=\frac{N(N-1)}{2}$. In bulk diamond, $T_{2}$ is approximately inversely proportional to the ${ }^{13} \mathrm{C}$ nuclear spin density $\eta=\frac{N}{V}$ as observed in Table 1 ; therefore, $T_{2} \sim \frac{1}{N} \sim \frac{1}{\sqrt{2 N_{\text {pair }}}}$. For an arbitrary nuclear spin $l$, the total number of flip-flop channels is $N_{\mathrm{cn}}=(2 /)^{2}$ and $T_{2} \sim \frac{1}{\sqrt{2 N_{\text {pair }} N_{\mathrm{cn}}}}$ giving $T_{2}(I) / T_{2}\left(I=\frac{1}{2}\right) \sim \frac{1}{2 l}$. This scaling relation is also useful to understand the role of quadrupole interactions (see Supplementary Information). For symmetrical electric field gradients, the quadrupole Hamiltonian shifts the spin sublevels with different $I_{z i}$ thus, only flip-flop transitions that swap the magnetic quantum numbers are allowed. In this case, $N_{\mathrm{cn}}=2 l$ and $T_{2}(I) / T_{2}\left(I=\frac{1}{2}\right) \sim \frac{1}{\sqrt{2 l}}$. Therefore, in the calculations on 2D systems presented below, neglecting the quadrupole term in the spin Hamiltonian leads to an underestimate of the calculated $T_{2}$.
Spin coherence in $\mathrm{MoS}_{2}$ and h-BN

We finally consider specific 2D materials as qubit hosts, namely $\mathrm{MoS}_{2}$ and $\mathrm{h}-\mathrm{BN}$. Both materials have large nuclear spin concentrations $\left(>25 \%\right.$ in $\mathrm{MoS}_{2}$ and $100 \%$ in $\left.\mathrm{h}-\mathrm{BN}\right)$, multiple nuclear species, and large nuclear spins. Suitable defects have not yet been identified in $\mathrm{MoS}_{2}$ and h-BN and here we consider mostly hypothetical defects with the same electron spin state $S=1$ and $\hat{z}$ direction of the magnetic axis as in the diamond NV center. The magnetic field is applied along the magnetic easy axis to maximize $T_{2}{ }^{21,38}$ Below, we present results for $\mathrm{h}-\mathrm{BN}$, with a nitrogen-substitution-vacancy complex $\mathrm{N}_{B} \mathrm{~V}_{\mathrm{N}}$ where the qubit is at the vacancy site, and for $\mathrm{MoS}_{2}$, where the qubit is located at a molybdenum site. We also considered qubits located at the boron site in h-BN and at the sulfur site in $\mathrm{MoS}_{2}$, and we found minor variations of $T_{2}(\sim 3 \%)$. The similarity of $T_{2}$ for different defects in the same host material is not surprising, as $T_{2}$ is determined by hundreds of nuclear spins and the effect of the local structure is negligible as long as the defect is a deep center with localized wavefunctions. We report below results for spin $S=1$, but we also considered the case of spin $S=\frac{1}{2}$, where we used the $\left|\frac{1}{2}\right\rangle$ and $\left|-\frac{1}{2}\right\rangle$ as the two-level system. Despite a minor change $(\sim 10 \%)$ in the numerical value of $T_{2}$, we found the same trends for spin $S=\frac{1}{2}$ and $S=1$.

As multiple nuclear spin species are present in both h-BN and $\mathrm{MoS}_{2}$, we first investigated the coupling of the various spin baths in the material. The spin coherence functions from each nuclear spin bath are shown in Fig. 2a, c. For non-interacting nuclear spin species, the total spin coherence function $\mathcal{L}$ can be written as a product $\mathcal{L}=\Pi_{a} \mathcal{L}_{a}$, where $\mathcal{L}_{a}$ is the coherence function from the $a$ th nuclear spin species. In Fig. 2b, d, we show that the computed $\mathcal{L}$ coincides with the one obtained from the product of the functions of each species, proving that different nuclear spin baths are decoupled. The reason for this decoupling is that the heteronuclear-spin flip-flop transitions are greatly suppressed in the
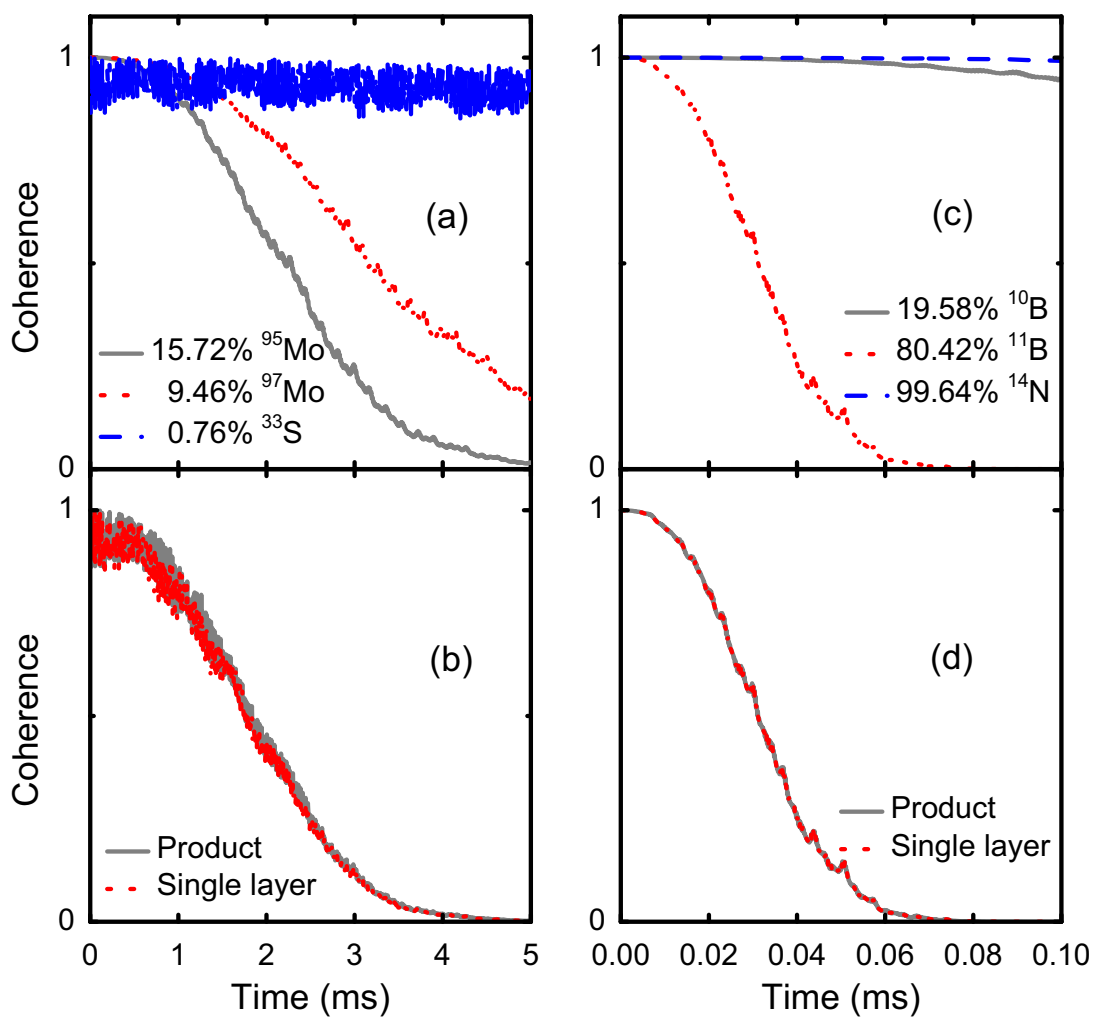

Fig. 2 Spin coherence functions computed for single-layer $\mathrm{MoS}_{2}$ (left panels) and h-BN (right panels). The upper panels show results for different spin baths separately. The lower panels compare results obtained for the total coherence function computed directly (red curve) and as a product (gray curve) of the coherence functions of the various spin baths, showing that the baths are decoupled 
Table 2. Computed coherence time $T_{2}$ (ms) for multi-layer and bulk $\mathrm{h}$ $\mathrm{BN}$ and $\mathrm{MoS}_{2}$ for natural abundance nuclear concentrations

\begin{tabular}{lllll}
\hline Materials & Bulk & 3-layer & 2-layer & 1-layer \\
\hline MoS $_{2}$ & 1.18 & 1.47 & 1.70 & 2.19 \\
h-BN & 0.018 & 0.024 & 0.028 & 0.036 \\
\hline
\end{tabular}

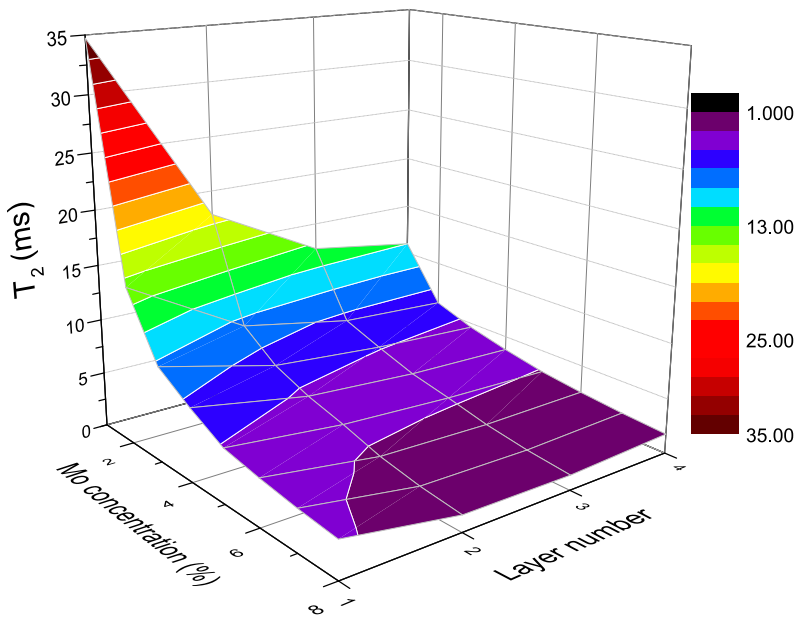

Fig. 3 Computed coherence time $T_{2}$ (ms) for multi-layer $\mathrm{MoS}_{2}$ as a function of Mo concentration. Here both ${ }^{95} \mathrm{Mo}$ and ${ }^{97}$ Mo have the same concentrations represented by the Mo concentration axis and the concentration of ${ }^{33} \mathrm{~S}$ is $0.76 \%$, corresponding to its natural abundance

presence of large magnetic fields because of the different gyromagnetic ratios from different nuclear spin species. A similar bath decoupling effect has also been observed in $\mathrm{SiC}^{44,45}$

Second, we considered the layer dependence of central spin decoherence in multi-layer $\mathrm{MoS}_{2}$ and $\mathrm{h}-\mathrm{BN}$ in their natural abundance nuclear bath (see Table 2). The magnetic field used in the simulation is $0.1 \mathrm{~T}$ for $\mathrm{MoS}_{2}$ and $0.5 \mathrm{~T}$ for $\mathrm{h}-\mathrm{BN}$, which have been tested to be in the strong field limit (see Supplementary Information). The $T_{2}$ increases with decreasing layer numbers, but there is only a two-fold enhancement for a single layer compared to the bulk. This insensitivity of $T_{2}$ to the number of layers is due to the large concentration of nuclear spins in both materials (see Fig. 1 ), and we would expect a more prominent layer dependence of $T_{2}$ in isotopic purified $\mathrm{MoS}_{2}$.

In $\mathrm{MoS}_{2}$, the concentration of ${ }^{33} \mathrm{~S}$ is negligible compared to that of Mo isotopes, and the decoherence of the electron spin mainly originates from the presence of ${ }^{95} \mathrm{Mo}$ and ${ }^{97} \mathrm{Mo}$ nuclear spins, as shown in Fig. 2a. We note that nuclear-spin-free Mo isotopes are commercially available. Therefore, we considered isotopically purified Mo and computed $T_{2}$ in single-layer, multi-layer, and bulk $\mathrm{MoS}_{2}$. The results at different nuclear spin concentrations are summarized in Fig. 3 (more details in the Supplementary Information). As the nuclear spin concentration decreases, the enhancement of $T_{2}$ is very large, especially for a single layer: at $1 \%$ ${ }^{95} \mathrm{Mo}, 1 \%{ }^{97} \mathrm{Mo}$, and $0.76 \%{ }^{33} \mathrm{~S}$, the single layer $T_{2}$ is 16 times larger than that computed for natural abundance concentration, while the $T_{2}$ of the bulk shows a six-fold increase. In other words, isotopic purification is a more effective tool in $2 \mathrm{D}$ than in $3 \mathrm{D}$ materials to engineer an enhancement of $T_{2}$. By further isotopic purification, the $T_{2}$ of a single layer $\mathrm{MoS}_{2}$ can exceed $30 \mathrm{~ms}$, an exceptionally long coherence time. In addition, we notice that the nuclear spin bath with $5 \%{ }^{95} \mathrm{Mo}$ and $5 \%{ }^{97} \mathrm{Mo}$ has a longer $T_{2}$ than the one with $10 \%{ }^{95} \mathrm{Mo}$ (see Supplementary Information), despite the similarity in the total nuclear spin concentrations. This is because the gyromagnetic ratios of ${ }^{95} \mathrm{Mo}$ and ${ }^{97} \mathrm{Mo}$ are similar (they differ by only $2 \%$ ), but at large magnetic field, their mutual flip-flops are suppressed. Therefore, the presence of multiple nuclear spin species offers a larger parameter space for isotopic engineering, and in turn this allows for the realization of longer $T_{2}$ without the need of eliminating all nuclear spins, which can then be used as additional quantum resources.

Figure $2 \mathrm{c}$ shows that in h-BN the decoherence is dominated by the ${ }^{11} \mathrm{~B}$ nuclear spin bath. This is due to the high concentration of ${ }^{11} \mathrm{~B}$ compared to ${ }^{10} \mathrm{~B}$, and, most importantly, to the much larger nuclear gyromagnetic ratio of ${ }^{11} \mathrm{~B}$ relative to ${ }^{10} \mathrm{~B}$ and ${ }^{14} \mathrm{~N}$. Hence, isotope engineering of $T_{2}$ in $\mathrm{h}-\mathrm{BN}$ should be focused on reducing ${ }^{11} \mathrm{~B}$ nuclear spin concentration. The total nuclear spin concentration from $\mathrm{B}$ nuclei cannot be changed, as $\eta\left({ }^{10} \mathrm{~B}\right)+\eta\left({ }^{11} \mathrm{~B}\right)=1$; therefore, we varied the relative concentration between $\eta\left({ }^{10} \mathrm{~B}\right)$ and $\eta\left({ }^{11} \mathrm{~B}\right)$. We found that $T_{2}$ increases monotonically as the ${ }^{10} \mathrm{~B}$ concentration increases, from $0.036 \mathrm{~ms}$ at $20 \%$ to $0.086 \mathrm{~ms}$, when all ${ }^{11} \mathrm{~B}$ nuclei are replaced by ${ }^{10} \mathrm{~B}$ nuclei.

\section{Effect of gyromagnetic ratio}

For spin natural abundance (see Table 2), the coherence time of $\mathrm{MoS}_{2}$ is almost two orders of magnitude longer than that of h-BN. The difference in nuclear spin concentration is not the only reason determining the large difference in $T_{2}$ : indeed at $100 \%{ }^{95} \mathrm{Mo}$, the difference between the $T_{2}$ of the two materials is still about one order of magnitude (see Supplementary Information). The difference in gyromagnetic ratios between ${ }^{11} \mathrm{~B}$ and ${ }^{95} \mathrm{Mo}$ is another important factor. As $\gamma\left({ }^{11} \mathrm{~B}\right) / \gamma\left({ }^{95} \mathrm{Mo}\right) \approx 5$, and the nuclear spin dipolar interaction $H_{\mathrm{nn}}$ (see Eq. 3) is proportional to $\gamma^{2}$, the difference in gyromagnetic ratios accounts for roughly 25 -fold enhancement of $T_{2}$ in $\mathrm{MoS}_{2}$ compared to h-BN. The small gyromagnetic ratios of ${ }^{95} \mathrm{Mo}$ and ${ }^{97} \mathrm{Mo}$ is also responsible for $T_{2}$ of bulk $\mathrm{MoS}_{2}$ with more than $20 \%$ nuclear spins being longer than that of diamond with only $1.1 \%$ nuclear spins. Many nuclei have a smaller gyromagnetic ratio than ${ }^{13} \mathrm{C}$ and compounds containing such nuclei may potentially be host materials with long coherence times.

In summary, we revealed, through simulations, the role of dimensionality and crystal geometry in controlling the decoherence of spin qubits in solids, which is as important as nuclear spin concentration. A key result of our study is that isotopic purification is much more effective in 2D than in conventional 3D materials such as $\mathrm{Si}$ and $\mathrm{C}$. We also demonstrated that in 2D, long spincoherence times may be engineered in solids with multi-nuclearspin environments, without sacrificing nuclear spins. In addition, our study provided a quantitative understanding of the influence of high nuclear spin baths $(I>1 / 2)$ on the spin coherence time $T_{2}$ of a defect-based spin qubit, and we identified the role of multiple physical parameters in controlling the qubit decoherence; such identification is critical in searching for promising host materials for spin qubits. Overall, we predicted that single-layer $\mathrm{MoS}_{2}$ layers, delta-doped diamond slabs, and $\mathrm{Si}$ thin slabs can be promising hosts for spin qubits, while the $T_{2}$ of $\mathrm{h}$-BN appears to be much more challenging to engineer, due to the large gyromagnetic ratio of the boron nuclei. In particular, we showed that $T_{2}$ of an isotopically purified layer of $\mathrm{MoS}_{2}$ is of the order of $30 \mathrm{~ms}$.

\section{METHODS}

Using the spin Hamiltonian $H$ (Eqs. 1-4) and the cluster-correlation expansion method, ${ }^{39}$ we computed the coherence function $\mathcal{L}=\frac{\left.\operatorname{Tr} \rho(t) \boldsymbol{S}_{1}\right]}{\operatorname{Tr} \rho(0) \boldsymbol{s}_{+}}$ for an ensemble of electron qubits with randomly distributed nuclear spins, where $\rho$ is the density operator and $\boldsymbol{S}_{+}$is the electron spin raising operator. The time $T_{2}$ was obtained from the profile of the ensemble averaged coherence function using $\mathcal{L}=e^{-\left(t / T_{2}\right)^{n}}$, where $n$ is the stretching exponent. Numerical convergence tests are reported in the Supplementary Information. 


\section{DATA AVAILABILITY}

The data that support the findings of this study are available upon request to the corresponding author.

\section{CODE AVAILABILITY}

The codes that were used in this study are available upon request to the corresponding author.

\section{ACKNOWLEDGEMENTS}

We thank David Awschalom, Chris Anderson, Gary Wolfowicz, Alexandre Bourassa, and Grant Smith for many useful discussions. This work was supported by the National Science Foundation (NSF) through the University of Chicago MRSEC under award number DMR-1420709. H.S. was also supported by the National Research Foundation of Korea (NRF) grant funded by the Korea government (MSIT) (No. 2018R1C1B6008980 and No. 2018R1A4A1024157).

\section{AUTHOR CONTRIBUTIONS}

M.Y. developed the numerical simulations and performed the theoretical calculations. H.S. and G.G. supervised the project. All authors contributed to the data analysis and production of the manuscript

\section{ADDITIONAL INFORMATION}

Supplementary Information accompanies the paper on the npj Computational Materials website (https://doi.org/10.1038/s41524-019-0182-3).

Competing interests: The authors declare no competing interests.

Publisher's note: Springer Nature remains neutral with regard to jurisdictional claims in published maps and institutional affiliations.

\section{REFERENCES}

1. Weber, J. R. et al. Quantum computing with defects. Proc. Natl. Acad. Sci. USA 107, 8513-8518 (2010).

2. Gruber, A. et al. Scanning confocal optical microscopy and magnetic resonance on single defect centers. Science 276, 2012-2014 (1997).

3. Jelezko, F. et al. Observation of coherent oscillation of a single nuclear spin and realization of a two-qubit conditional quantum gate. Phys. Rev. Lett. 93, 130501 (2004).

4. Koehl, W. F. et al. Room temperature coherent control of defect spin qubits in silicon carbide. Nature 479, 84 (2011).

5. Baranov, P. G. et al. Silicon vacancy in sic as a promising quantum system for single-defect and single-photon spectroscopy. Phys. Rev. B 83, 125203 (2011).

6. Siyushev, P. et al. Optically controlled switching of the charge state of a single nitrogen-vacancy center in diamond at cryogenic temperatures. Phys. Rev. Lett 110, 167402 (2013).

7. Von Bardeleben, H. J., Cantin, J. L., Rauls, E. \& Gerstmann, U. Identification and magneto-optical properties of the nv center in $4 \mathrm{~h}$ - sic. Phys. Rev. B 92, 064104 (2015).

8. Zwanenburg, F. A. et al. Silicon quantum electronics. Rev. Mod. Phys. 85, 961 (2013).

9. Xia, K. et al. All-optical preparation of coherent dark states of a single rare earth ion spin in a crystal. Phys. Rev. Lett. 115, 093602 (2015).

10. Jiang, Q.-D. Wilczek, F. Quantum atmospherics for materials diagnosis. Preprint at arXiv:1809.01692 (2018).

11. Degen, C. L., Reinhard, F. \& Cappellaro, P. Quantum sensing. Rev. Mod. Phys. 89 035002 (2017a).

12. Hahn, E. L. Spin echoes. Phys. Rev. 80, 580 (1950).

13. DiVincenzo, D. P. The physical implementation of quantum computation. Fortschr Phys 48, 771-783 (2000).

14. Graham, M. J., Zadrozny, J. M., Fataftah, M. S. \& Freedman, D. E. Forging solid-state qubit design principles in a molecular furnace. Chem. Mater. 29, 1885-1897 (2017).

15. Degen, C. L., Reinhard, F. \& Cappellaro, P. Quantum sensing. Rev Mod. Phys. 89, 035002 (2017b).

16. Myers, B. A. et al. Probing surface noise with depth-calibrated spins in diamond Phys. Rev. Lett. 113, 027602 (2014).
17. Kim, M. et al. Decoherence of near-surface nitrogen-vacancy centers due to electric field noise. Phys. Rev. Lett. 115, 087602 (2015).

18. de Sousa, R. \& Sarma, S. D. Theory of nuclear-induced spectral diffusion: spin decoherence of phosphorus donors in si and gaas quantum dots. Phys. Rev. B 68 115322 (2003).

19. Balasubramanian, G. et al. Ultralong spin coherence time in isotopically engineered diamond. Nat. Mater. 8, 383 (2009).

20. Tyryshkin, A. M. et al. Electron spin coherence exceeding seconds in high-purity silicon. Nat. Mater. 11, 143 (2012).

21. Stanwix, P. L. et al. Coherence of nitrogen-vacancy electronic spin ensembles in diamond. Phys. Rev. B 82, 201201 (2010).

22. Xia, F. et al. Two-dimensional material nanophotonics. Nat. Photonics 8 , 899 (2014).

23. Liu, X. et al. Strong light-matter coupling in two-dimensional atomic crystals. Nat Photonics 9, 30 (2015).

24. $\mathrm{Wu}, \mathrm{S}$. et al. Monolayer semiconductor nanocavity lasers with ultralow thresholds. Nature 520, 69 (2015).

25. Majumdar, A. et al. Hybrid 2d material nanophotonics: a scalable platform for low-power nonlinear and quantum optics. ACS Photonics 2, 1160-1166 (2015).

26. Lin, Z. et al. Defect engineering of two-dimensional transition metal dichalcogenides. 2D Mater. 3, 022002 (2016).

27. Tran, T. T. et al. Quantum emission from hexagonal boron nitride monolayers. Nat. Nanotechnol. 11, 37 (2016).

28. Tran, T. T. et al. Robust multicolor single photon emission from point defects in hexagonal boron nitride. ACS Nano 10, 7331-7338 (2016).

29. Jungwirth, N. R. et al. Temperature dependence of wavelength selectable zerophonon emission from single defects in hexagonal boron nitride. Nano Lett. 16 6052-6057 (2016).

30. Kianinia, M. et al. All-optical control and super-resolution imaging of quantum emitters in layered materials. Nat. Commun. 9, 874 (2018).

31. Grosso, G. et al. Tunable and high-purity room temperature single-photon emission from atomic defects in hexagonal boron nitride. Nat. Commun. 8, 705 (2017).

32. Exarhos, A. L. et al. Magnetic-field-dependent quantum emission in hexagonal boron nitride at room temperature. Nat. Commun. 10, 222 (2019).

33. Palacios-Berraquero, C. et al. Large-scale quantum-emitter arrays in atomically thin semiconductors. Nat. Commun. 8, 15093 (2017).

34. Tonndorf, P. et al. Single-photon emission from localized excitons in an atomically thin semiconductor. Optica 2, 347-352 (2015).

35. Zhang, S. et al. Defect structure of localized excitons in a wse 2 monolayer. Phys. Rev. Lett. 119, 046101 (2017).

36. Witzel, W. M. \& Sarma, S. D. Quantum theory for electron spin decoherence induced by nuclear spin dynamics in semiconductor quantum computer architectures: spectral diffusion of localized electron spins in the nuclear solid-state environment. Phys. Rev. B 74, 035322 (2006).

37. Yao, W., Liu, R.-B. \& Sham, L. J. Theory of electron spin decoherence by interacting nuclear spins in a quantum dot. Phys. Rev. B 74, 195301 (2006).

38. Maze, J. R., Taylor, J. M. \& Lukin, M. D. Electron spin decoherence of single nitrogen-vacancy defects in diamond. Phys. Rev. B 78, 094303 (2008).

39. Yang, W. \& Liu, R.-B. Quantum many-body theory of qubit decoherence in a finite-size spin bath. Phys. Rev. B 78, 085315 (2008).

40. Schweiger, A. \& Jeschke, G. Principles of Pulse Electron Paramagnetic Resonance (Oxford University Press, Oxford, 2001).

41. Unden, T. et al. Coherent control of solid state nuclear spin nano-ensembles. $n p j$ Quantum Inform. 4, 39 (2018).

42. Slota, M. et al. Magnetic edge states and coherent manipulation of graphene nanoribbons. Nature 557, 691 (2018).

43. Zhao, N., Ho, S.-W. \& Liu, R.-B. Decoherence and dynamical decoupling control of nitrogen vacancy center electron spins in nuclear spin baths. Phys. Rev. B 85, 115303 (2012).

44. Seo, H. et al. Quantum decoherence dynamics of divacancy spins in silicon carbide. Nat Commun. 7, 12935 (2016).

45. Yang, L.-P. et al. Electron spin decoherence in silicon carbide nuclear spin bath. Phys. Rev. B 90, 241203 (2014).

46. Ono, K., Mori, T. M. \& Moriyama, S. High-temperature operation of a silicon qubit Sci. Rep. 9, 469 (2019).

47. Nylandsted Larsen, A. et al. Tin-vacancy acceptor levels in electron-irradiated ntype silicon. Phys. Rev. B 62, 4535 (2000).

48. Zhao, N., Ho, S.-W. \& Liu, R.-B. Decoherence and dynamical decoupling control of nitrogen vacancy center electron spins in nuclear spin baths. Phys. Rev. B 85 115303 (2012).

49. Hall, L. T., Cole, J. H. \& Hollenberg, L. C. L. Analytic solutions to the central-spin problem for nitrogen-vacancy centers in diamond. Phys. Rev. B 90, 075201 (2014). 
Open Access This article is licensed under a Creative Commons Attribution 4.0 International License, which permits use, sharing, adaptation, distribution and reproduction in any medium or format, as long as you give appropriate credit to the original author(s) and the source, provide a link to the Creative Commons license, and indicate if changes were made. The images or other third party material in this article are included in the article's Creative Commons license, unless indicated otherwise in a credit line to the material. If material is not included in the article's Creative Commons license and your intended use is not permitted by statutory regulation or exceeds the permitted use, you will need to obtain permission directly from the copyright holder. To view a copy of this license, visit http://creativecommons. org/licenses/by/4.0/.

(c) The Author(s) 2019 